\title{
Causas de muerte de pacientes con insuficiencia cardíaca con fracción de eyección reducida en seguimiento por un programa multidisciplinario
}

Silvana Estrada Escobar ORCID: 0000-0002-5505-4642

Postgrado Medicina Interna. Clínica Médica A. Hospital Clínicas.

Pablo Álvarez Rocha ORCID: 0000-0002-4550-3483 Internista. Cardiólogo. Profesor Agregado Clínica Médica A. Hospital

Clínicas.

Lucía Florio Legnani ORCID: 0000-0002-9608-2666 Cardiólogo. Profesor Agregado Cátedra de Cardiología. Hospital

Clínicas.

Gabriela Ormaechea Gorricho

ORCID: 0000-0002-2981-7722 Internista. Profesor Director Clínica

Médica A. Hospital Clínicas

Grupo UMIC: Acle, Santiago Albistur, Sebastián; Álvarez, Flavia; Álvarez, Pablo; Andrade, Rodrigo; Carlevaro, Lía; Chamorro, Cristina;

Florio, Lucia; Freire, Diego; Gómez, Andreina; Oliva, Leonardo;

Ormaechea, Gabriela; Parma,

Gabriel; Perez, Verónica; Ramos,

Camila; Rojas, Leticia; Silvera, Gabriela; Trelles, Victoria; Zeballos, Jacqueline.

\section{Causes of death of patients with heart failure with reduced ejection fraction in follow-up by a multidisciplinary program}

Causas de morte de pacientes com insuficiência cardíaca com fração de ejeção reduzida no seguimento de um programa multidisciplinar

Resumen: Introducción: La Insuficiencia cardiaca es una patología con alta prevalencia, con sobrevida menor a $50 \%$ a 4 años en los estadios avanzados. En Uruguay existen pocos datos sobre su morbimortalidad. La unidad multidisciplinaria de insuficiencia cardiaca del Hospital de Clínicas (UMIC) desarrolla un programa de seguimiento a través de una policlínica especializada. El objetivo de este estudio es conocer las causas de muerte de la cohorte UMIC y analizar posibles factores asociados a ellas.

Material y métodos: Estudio descriptivo, observacional, de seguimiento de cohorte retrospectivo. Se constató el estado vital de los pacientes admitidos y registrados desde octubre del 2003 hasta septiembre de 2016 mediante recolección pasiva y activa de datos. El grado de certificación fue a través del certificado de defunción. Se analizaron características de los pacientes fallecidos, Se diferenciaron causas de muerte en cardiacas y no cardiacas y se analizaron predictores asociados a dichas causas. Resultados: En un total de 1041 pacientes, se registraron 182 muertes en el período estudiado. La media de edad fue 69 \pm 5 años con predominio del sexo masculino (3/1). Mediana de sobrevida de 2,6 años. En 99 pacientes (61\%) se documentó muerte cardíaca y de ellos $35 \%$ por muerte súbita, $56 \%$ insuficiencia cardiaca y $8 \%$ infarto agudo de miocardio fatal. Las causas no cardíacas fueron infecciosas en $59 \%$, neoplásicas $18 \%$, por accidente cerebrovascular $14 \%$ y por otras causas $8 \%$. Se encontró asociación positiva de presencia de neoplasia con muerte no cardiaca (OR 7,56; IC 95\% 1,93-29,67; p=0,004). Conclusiones: Primera publicación que describe causas de muerte en una cohorte de pacientes con IC en Uruguay con una tasa de mortalidad anual de 5,6 \% con alta incidencia de muertes no cardiacas. La presencia de neoplasia se asoció a mayor mortalidad de causa no cardiaca.

Palabras clave: Insuficiencia cardiaca; muerte; mortalidad; programas de seguimiento

Abstract: Introduction: Heart failure is a pathology with high prevalence. In advanced stages its survival is less than $50 \%$ at 4 years. Few data are available about morbidity and mortality in Uruguay. The multidisciplinary unit of heart failure at Hospital de Clínicas (UMIC) develops a management program through a specialized out-patient service. The objective of this study is to know causes of death of the UMIC cohort and analyze possible associated factors. Material and methods: Descriptive, observational, retrospective cohort study. The vital status of the patients admitted and registered from October 2003 to September 2016 was verified through passive and active data collection. The degree of certification was established through the death certificate. Characteristics of death subjects were analyzed. Causes of death were differentiated in cardiac and non-cardiac and predictors associated to the different causes of death were analyzed. Results: From 1041 patients, 182 deaths were recorded in the period studied. The average age was $69 \pm 5$ years with males 'predominance (3/1). Median survival was 2.6 years since recruitment. In 99 patients (61\%), cardiac death was documented, $35 \%$ of them as sudden death, $56 \% \mathrm{Cl}$ and 
$8 \%$ fatal myocardial infarction. Non-cardiac causes were infectious in $59 \%$, neoplasia in $18 \%$, stroke in $14 \%$ and other causes in $8 \%$. A positive association was found between the presence of neoplasia and non-cardiac death (OR 7.56, 95\% Cl 1.93-29.67, $p=0.004)$. Conclusions: This is a first publication describing causes of death in a cohort of patients with HF in Uruguay with an annual mortality rate of $5.6 \%$ with a high incidence of non-cardiac deaths. The presence of neoplasia was associated with higher non-cardiac mortality.

Key words: Heart failure; death; mortality; tracking programs

Resumo: Introdução: A insuficiência cardíaca é uma patologia com alta prevalência, com sobrevida inferior a $50 \%$ aos 4 anos nos estágios avançados. No Uruguai, existem poucos dados sobre sua morbimortalidade. A unidade multidisciplinar de insuficiência cardíaca do Hospital de Clínicas (UMIC) desenvolve um programa de acompanhamento por meio de uma policlínica especializada. O objetivo deste estudo é conhecer as causas de morte da coorte UMIC e analisar possíveis fatores associados a elas. Material e métodos: Estudo de acompanhamento de coorte descritivo, observacional e retrospectivo. O status vital dos pacientes admitidos e registrados de outubro de 2003 a setembro de 2016 foi verificado por meio de coleta de dados passiva e ativa. O grau de certificação foi através do atestado de óbito. As características dos pacientes falecidos foram analisadas, as causas de morte cardíaca e não cardíaca foram diferenciadas e os preditores associados a essas causas foram analisados. Resultados: No total de 1041 pacientes, foram registradas 182 mortes no período estudado. A idade média foi de 69 \pm 5 anos, com predominância masculina (3/1). Sobrevida mediana de 2,6 anos. Em 99 pacientes (61\%), a morte cardíaca foi documentada e $35 \%$ deles por morte súbita, $56 \%$ de insuficiência cardíaca e $8 \%$ de infarto agudo do miocárdio fatal. Causas não cardíacas foram infecciosas em $59 \%$, neoplásicas $18 \%$, devido a acidente vascular cerebral $14 \%$ e outras causas $8 \%$. Foi encontrada associação positiva na presença de neoplasia com morte não cardíaca (OR 7,56; IC 95\% 1,93-29,67; $p=0,004)$. Conclusões: Primeira publicação descrevendo causas de morte em uma coorte de pacientes com IC no Uruguai, com uma taxa de mortalidade anual de 5,6\%, com alta incidência de mortes não cardíacas. A presença de neoplasia foi associada a maior mortalidade por causas não cardíacas.

Palavras-chave: Insuficiência cardíaca; morte mortalidade; programas de rastreamento 


\section{Introducción}

La Insuficiencia cardiaca (IC) es un problema sanitario con alta y creciente prevalencia debido al mayor envejecimiento poblacional y a la mayor sobrevida de pacientes con patologías cardiovasculares que transitan hacia la IC como vía final común. ${ }^{(1,2)}$

El pronóstico de la IC es malo con cifras de supervivencia a 4 años inferiores al $50 \%$ para la IC avanzada, siendo las principales causas de muerte la muerte súbita (MS) y la falla de bomba refractaria. ${ }^{(3)}$ Distintos registros muestran que una parte importante de los pacientes con IC fallecen por procesos ajenos a la misma, en especial por neoplasias, patología respiratoria y septicemias. ${ }^{(4)}$

Existen pocos datos sobre la morbimortalidad de esta enfermedad en el Uruguay. La Comisión Honoraria para la Salud Cardiovascular utiliza los egresos hospitalarios por enfermedades del sistema circulatorio como aproximación a la medida de morbilidad; la cardiopatía isquémica y la IC son las causas más frecuentes de egreso. ${ }^{(5)}$

La unidad multidisciplinaria de IC del Hospital de Clínicas (UMIC) desarrolla su tarea desde el año 2003 con la concepción de programa de seguimiento de enfermedades crónicas y el método de policlínica especializada. Recibe la mayor parte de los pacientes desde los servicios de cardiología, de medicina interna, policlínicas periféricas de asistencia primaria y secundaria y un menor porcentaje proviene de emergencia.

El objetivo de este estudio es conocer las causas de muerte de la población asistida en la UMIC y analizar posibles factores asociados a dichas causas.

\section{Material y métodos}

Se trata de un estudio descriptivo, observacional, con seguimiento de cohorte retrospectivo.

Se incluyen todos los pacientes admitidos en la UMIC en el período comprendido entre octubre de 2003 y setiembre de 2016, registrando muerte como evento y la causa de la misma para el análisis. Los criterios de inclusión a la cohorte UMIC son: edad mayor a 18 años y ser portador de IC con fracción de eyección del ventrículo izquierdo (FEVI) reducida (ICFEr) (FEVI < $40 \%$ ) de cualquier causa. En los casos en que exista remodelación reversa, con recuperación de la FEVI, los pacientes no se excluyen.

La situación vital se constató mediante recolección pasiva de datos de las historias clínicas y recolección activa por contacto con familiares o cuidadores de los fallecidos, así como también con los centros sanitarios donde hubieran estado internados al momento de la muerte. La certificación de la muerte fue, en todos los casos, a través del certificado de defunción obtenido de la División de Epidemiologia del Ministerio de Salud, asignando causas de muerte según el código CIE 10 de la OMS. ${ }^{(6)}$

Se analizaron distintas variables en las características de los fallecidos tales como comorbilidades, antecedentes personales, marcadores funcionales y humorales, buscando posibles asociaciones con la causa de muerte. Las variables dinámicas se tipificaron de acuerdo a la constancia en el último registro antes del fallecimiento.

Se diferencian las causas de muerte en cardiacas y no cardiacas; se definieron como causas cardiacas la MS, el infarto agudo de miocardio fatal (IAM) y la IC, y como causas no cardiacas cualquier otra diferente a las definidas como cardiacas.

La UMIC cuenta con un consentimiento informado para todos los pacientes que se asisten en ella mediante el cual autorizan a la utilización de sus datos clínicos para investigación. El mismo fue aprobado por el comité de ética del Hospital de Clínicas.

\section{Análisis estadístico}

Las variables continuas se expresan como media \pm desvío estándar (DE) o mediana [intervalo intercuartílico], según tengan o no distribución normal; las variables categóricas se presentan como porcentajes. Se calculó la tasa anual de mortalidad.

Se realizó un análisis univariado y multivariable mediante regresión logística con muerte cardíaca y no cardíaca como variable dependiente. Las curvas de sobrevida se analizan con gráfica de Kaplan Meier y log rank. 
La significación estadística se estableció en $\mathrm{p}<0,05$.

\section{Resultados}

Se documentaron 182 muertes en un total de 1041 pacientes registrados correspondiendo a una incidencia relativa de $17,5 \%$ en 13 años y a una incidencia absoluta de 13,9 muertes por año. La tasa anual de mortalidad fue 5,6 cada 100 pacientes/año.

Hubo 98 pacientes del registro perdidos de vista. No se pudo acceder a la causa de muerte de 22 pacientes. Debido a una migración de datos realizada de la base inicial a otra se perdieron datos parciales de 54 de los pacientes fallecidos con causa de muerte conocida por lo cual el análisis descriptivo se realiza sobre un total de 128 pacientes.

La media de edad fue $69 \pm 5$ años, con predominio del sexo masculino sobre el femenino (3/1). En la tabla 1 se presentan las características de la población fallecida.

\begin{tabular}{|l|l|l|l|l|l|}
\hline \multicolumn{7}{|c|}{ Características de la población (N=128) } \\
\hline Género & $\mathbf{n}(\%)$ & Comorbilidades & $\mathbf{n}(\%)$ & Tratamiento & $\mathbf{n}(\%)$ \\
\hline Masculino & $90(70)$ & HTA & $104(81)$ & BB & $109(85)$ \\
\hline Femenino & $38(30)$ & Dislipemia & $70(55)$ & IECA & $89(70)$ \\
\hline Etiología & $\mathbf{n}(\%)$ & FA & $53(41)$ & ARA II & $12(9)$ \\
\hline C. Isquémica & $60(47)$ & ERC & $47(37)$ & ARM & $13(10)$ \\
\hline C. no isquémica & $43(34)$ & Anemia & $38(30)$ & FEVI & n (\%) \\
\hline C. no definida & $25(19)$ & Diabetes & $37(29)$ & $\leq 29 \%$ & $54(42)$ \\
\hline CF (NYHA) & $\mathbf{n}(\%)$ & Tabaquismo & $36(28)$ & $30 \%-39 \%$ & $57(45)$ \\
\hline I & $20(16)$ & Alcoholismo & $23(18)$ & $\geq 49 \%$ & $17(17)$ \\
\hline II & $53(41)$ & Distiroidismos & $14(11)$ & & \\
\hline III & $45(35)$ & EPOC & $13(10)$ & & \\
\hline IV & $10(8)$ & Neoplasia & $11(9)$ & & \\
\hline \multicolumn{7}{|l|}{} & Asma & $3(2)$ & & \\
\hline Edad media \pm DE (años): $69 \pm 5$ & & & \\
\hline
\end{tabular}

La muerte de causa cardiaca se constató en 99 pacientes (61\%) correspondiendo 35\% (35 pacientes) a MS, $56 \%$ (56 pacientes) a IC y $8 \%$ (8 pacientes) a IAM.

Las causas de muerte no cardiaca (61 pacientes) correspondieron a causas infecciosas en 59\% (36 pacientes), neoplásicas 18\% (11 pacientes) accidente cerebrovascular 14\% (9 pacientes) y en $8 \%$ (5 pacientes) correspondiendo a diferentes otras causas.

En la tabla 2 y tabla 3 se presentan los análisis univariados de mortalidad cardíaca y no cardíaca respectivamente.

En el análisis univariado se encontró una fuerte asociación positiva de la presencia de neoplasia con la muerte no cardíaca (OR 5,29; IC95\% 1,48-18,83; p 0,01) y una asociación negativa de dicha presencia con la causa de muerte cardíaca (OR 0,24 con $\mathrm{p}<0,04)$.

\begin{tabular}{|l|l|l|l|}
\hline \multicolumn{5}{|c}{ Análisis univariado para muerte cardiaca } \\
\hline Variable & OR/Coef.. $\beta$ & IC $95 \%$ & $p$ \\
\hline Sexo & 1,36 & $0,72-2,56$ & 0,35 \\
\hline Edad & 0,01 & $-0,02-0,04$ & 0,34 \\
\hline CF (NYHA) & 1,45 & $0,95-2,23$ & 0,09 \\
\hline FEVI & 0,01 & $-0,03-0,05$ & 0,71 \\
\hline IMC & $-0,03$ & $-0,12-0,06$ & 0,48 \\
\hline C. Isquémica & 1,5 & $0,55-2,46$ & 0,33 \\
\hline Diabetes & 0,5 & $0,23-1,08$ & 0,08 \\
\hline HTA & 0,87 & $0,35-2,13$ & 0,75 \\
\hline Tabaquismo & 1 & $0,46-2,18$ & 0,99 \\
\hline
\end{tabular}


Tabla 2: Análisis univariado para muerte de causa cardiaca en 128 sujetos fallecidos de la cohorte UMIC.

CF $(N Y H A)=$ clase funcional según la New York Heart Association; HTA=hipertensión arterial; $F A=$ fibrilación auricular; $E R C=$ enfermedad renal crónica; EPOC= enfermedad pulmonar obstructiva crónica; $\mathrm{BB}=$ betabloqueantes; $\mathrm{IECA}=$ inhibidores de la enzima convertidora de angiotensina; ARA $\|=$ antagonistas de receptores de angiotensina II; ARM = antagonistas de receptores mineralocorticoideos; $\mathrm{FEVI}=$ fracción de eyección del ventrículo izquierdo; NTproBNP = porción aminoterminal de péptido natriurético $B$

Tabla 3: Análisis univariado para muerte de causa no cardiaca en 128 sujetos fallecidos de la cohorte UMIC. $\mathrm{CF}(\mathrm{NYHA})=$ clase funcional según la New York Heart Association; HTA=hipertensión arterial; $F A=$ fibrilación auricular; $E R C=$ enfermedad renal crónica; $\mathrm{EPOC}=$ enfermedad pulmonar obstructiva crónica; $\mathrm{BB}=$ betabloqueantes; $\mathrm{IECA}=$ inhibidores de la enzima convertidora de angiotensina; ARA $\|=$ antagonistas de receptores de angiotensina

II; ARM = antagonistas de receptores

mineralocorticoideos; $F E V l=$ fracción de eyección del ventrículo izquierdo; NTproBNP $=$ porción aminoterminal de péptido

\begin{tabular}{|l|l|l|l|}
\hline Dislipemia & 0,51 & $0,25-1,03$ & 0,06 \\
\hline Asma & 1,62 & $0,14-18,37$ & 0,67 \\
\hline Alcohol & 0,69 & $0,28-1,70$ & 0,42 \\
\hline ERC & 0,84 & $0,41-1,73$ & 0,64 \\
\hline Anemia & 0,49 & $0,22-1,01$ & 0,08 \\
\hline EPOC & 0,58 & $0,17-2,00$ & 0,39 \\
\hline NT proBNP & 0,00 & $-0,00-0,00$ & 0,61 \\
\hline Distiroidismos & 0,78 & $0,23-2,37$ & 0,66 \\
\hline Neoplasias & 0,24 & $0,06-0,91$ & 0,04 \\
\hline FA & 0,62 & $0,30-1,26$ & 0,19 \\
\hline Diurético & 1,02 & $0,39-2,67$ & 0,96 \\
\hline Betabloqueantes & 1 & $0,37-2,71$ & 0,99 \\
\hline IECA & 1,5 & $0,69-2,15$ & 0,31 \\
\hline ARA II & 0,78 & $0,24-2,58$ & 0,7 \\
\hline ARM & 0,46 & $0,14-2,31$ & 0,2 \\
\hline
\end{tabular}

\begin{tabular}{|l|l|l|l|}
\hline \multicolumn{5}{|c}{ Análisis univariado para muerte no cardiaca } \\
\hline Variable & OR/Coef. $\beta$ & IC 95\% & \multicolumn{1}{l|}{} \\
\hline Sexo & 0,8 & $0,41-1,56$ & 0,51 \\
\hline Edad & 0,00 & $-0,03-0,03$ & 0,87 \\
\hline CF & 0,72 & $0,45-1,14$ & 0,17 \\
\hline FEVI & $-0,01$ & $-0,05-0,04$ & 0,81 \\
\hline IMC & 0,08 & $-0,01-0,18$ & 0,09 \\
\hline C. Isquémica & 1,06 & $0,74-1,5$ & 0,76 \\
\hline Diabetes & 1,81 & $0,81-4,07$ & 0,15 \\
\hline HTA & 1,36 & $0,49-3,75$ & 0,56 \\
\hline Tabaquismo & 0,45 & $0,18-1,15$ & 0,1 \\
\hline Dislipemia & 2,2 & $1,0-4,80$ & 0,05 \\
\hline Asma & 1,1 & $0,01-12,56$ & 0,94 \\
\hline Alcohol & 0,6 & $0,2-1,75$ & 0,35 \\
\hline ERC & 1,66 & $0,76-3,58$ & 0,20 \\
\hline Anemia & 1,47 & $0,63-3,41$ & 0,37 \\
\hline EPOC & 1,43 & $0,4-5,15$ & 0,59 \\
\hline NT proBNP & $-0,00$ & $-0,00-0,00$ & 0,77 \\
\hline Distiroidismos & 2,47 & $0,8-7,60$ & 0,12 \\
\hline Neoplasias & 5,29 & $1,48-18,83$ & 0,01 \\
\hline FA & 1,83 & $0,85-4,0$ & 0,12 \\
\hline Diurético & 0,98 & $0,34-2,8$ & 0,97 \\
\hline Betabloqueantes & 1,7 & $0,52-5,55$ & 0,38 \\
\hline IECAs & 0,87 & $0,39-1,95$ & 0,73 \\
\hline ARA II & 1,11 & $0,31-3,95$ & \\
\hline ARM & 2,05 & $0,64-5,57$ & \\
\hline & & & 0,22 \\
\hline
\end{tabular}

En la tabla 4 y tabla 5 se presentan los análisis multivariados de mortalidad cardíaca y no cardíaca respectivamente.

Tabla 4: Análisis multivariado para muerte de causa cardíaca en 128 sujetos fallecidos de la cohorte UMIC. $\mathrm{CF}(\mathrm{NYHA})=$ clase funcional según la New York Heart Association.

\begin{tabular}{|l|l|l|l|}
\hline \multicolumn{4}{|c|}{ Regresión logística multivariada para muerte cardíaca } \\
\hline Variable & OR & IC $95 \%$ & p \\
\hline CF (NYHA) & 1,54 & $0,96-2,45$ & 0,07 \\
\hline Diabetes & 0,57 & $0,25-1,31$ & 0,19 \\
\hline Dislipemia & 0,53 & $0,25-1,13$ & 0,10 \\
\hline
\end{tabular}


Tabla 5: Análisis multivariado para muerte de causa no cardíaca en 128 sujetos fallecidos de la cohorte UMIC. York Heart Association.

Tabla 6: Análisis multivariado con regresión de Cox para muerte de causa cardíaca en 128 sujetos fallecidos de la cohorte UMIC. CF $(\mathrm{NYHA})=$ clase funcional según la New York Heart Association.

Tabla 7: Análisis multivariado con regresión de Cox para muerte de causa no cardíaca en 128 sujetos fallecidos de la cohorte UMIC. según la New York Heart Association.

\begin{tabular}{|l|l|l|l|}
\hline Anemia & 0,55 & $0,23-1,32$ & 0,18 \\
\hline Neoplasia & 0,23 & $0,05-0,97$ & 0,046 \\
\hline
\end{tabular}

\begin{tabular}{|l|l|l|l|}
\hline \multicolumn{4}{|c|}{ Regresión logística multivariada para muerte no cardíaca } \\
\hline Variable & OR/Coef. $\beta$ & {$[95 \%$ Cl] } & p \\
\hline Tabaquismo & 0,37 & $0,08-1,72$ & 0,20 \\
\hline Dislipemia & 2,80 & $0,77-10,13$ & 0,12 \\
\hline Neoplasia & 9,27 & $1,54-52,20$ & 0,01 \\
\hline IMC & 1.07 & $0,95-1,20$ & 0,26 \\
\hline
\end{tabular}

Para el análisis multivariado se tomaron todas las variables con una $p \leq 0,1$ en el univariado. En dicho análisis la neoplasia vuelve a mostrar la asociación estadísticamente significativa negativa y positiva para las causas de muerte cardiaca y no cardiaca respectivamente mientras que la clase funcional mostró una tendencia a asociar su peoría con la muerte cardiaca, pero sin alcanzar valores significativos.

En las tablas 6 y 7 se presentan los análisis multivariados con regresión de Cox para muerte cardíaca y no cardíaca respectivamente.

En los mismos se confirmó la asociación de neoplasia.

\begin{tabular}{|l|l|l|l|}
\hline \multicolumn{4}{|c|}{ Multivariado regresión de Cox para muerte cardíaca } \\
\hline Variable & HR & IC 95\% & p \\
\hline CF (NYHA) & 1,03 & $0,81-1,38$ & 0,64 \\
\hline Diabetes & 1,12 & $0,67-1,85$ & 0,65 \\
\hline Dislipemia & 0,62 & $0,39-0,97$ & 0,038 \\
\hline Anemia & 0,75 & $0,44-1,28$ & 0,30 \\
\hline Neoplasia & 0,54 & $0,19-1,50$ & 0,24 \\
\hline
\end{tabular}

\begin{tabular}{|l|l|l|l|}
\hline \multicolumn{4}{|c|}{ Multivariado regresión de Cox para muerte no cardíaca } \\
\hline Variable & HR & {$[95 \%$ Cl] } & P \\
\hline Tabaquismo & 0,82 & $0,25-2,64$ & 0,73 \\
\hline Dislipemia & 1,9 & $0,65-5,55$ & 0,23 \\
\hline Neoplasia & 4,3 & $1,58-12,09$ & 0,004 \\
\hline IMC & 1,02 & $0,94-1,10$ & 0,53 \\
\hline
\end{tabular}

La mediana de sobrevida sobre 128 observaciones válidas fue 973 días (2,6 años) y sin diferencias significativas en las curvas de sobrevida de aquellos con muerte cardíaca vs no cardíaca (log rank $p=0,81$ ). Figura 1 y Figura 2. 
Figura 1: Gráfico de Kaplan Meier de sobrevida estimada en 128 sujetos fallecidos de la cohorte UMIC. Mediana de sobrevida 973 días.

Figura 2: Gráfico de sobrevida de Kaplan Meier según causa de muerte en 128 sujetos fallecidos de la cohorte UMIC.
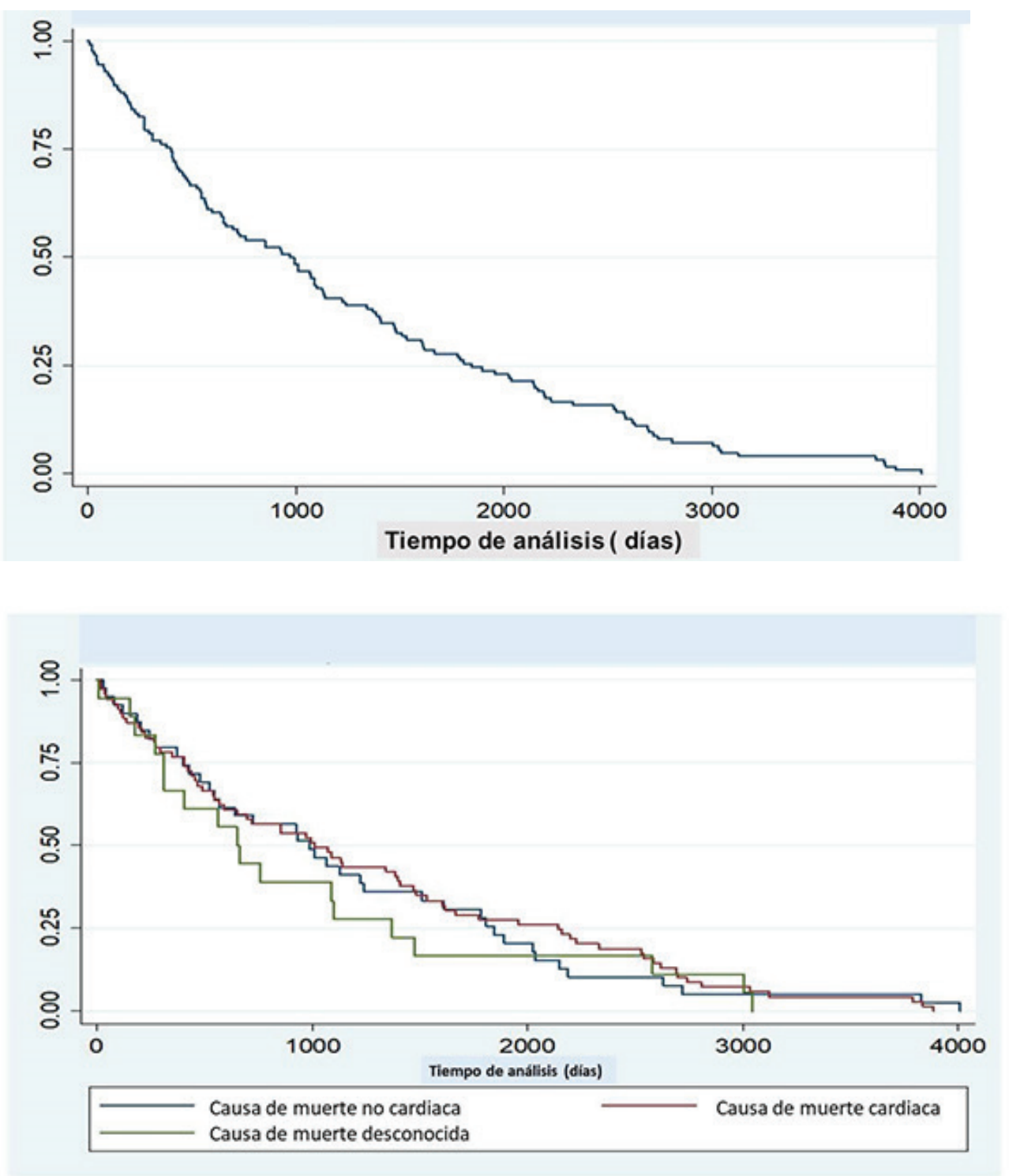

\section{Discusión}

Esta es la primera publicación que describe las causas de muerte en una cohorte de pacientes con IC en el Uruguay. La tasa de mortalidad fue 5,6 por cien pacientes/año. En América Latina es escasa la información sobre la carga de enfermedad y la mortalidad por IC; podría realizarse una aproximación a la mortalidad en la población general a través de la revisión de Ciaponi y cols con datos poblacionales de Brasil que evidencian una mortalidad de 168/100000 personas/año. (7) De todas maneras, nuestros datos se restringen a una población particular y se deben comparar con registros similares. Pons y cols, en una unidad especializada española con una población similar a la nuestra hallaron una mortalidad, en el primer año, de 10\% con una acumulada a los 7 años de $50 \%$ aproximadamente; muy superior, por cierto, a la hallada en nuestro registro. ${ }^{(8)}$

Sin embargo, la tasa anual de mortalidad obtenida en nuestro registro se asemeja a registros europeos. ${ }^{(4,9)}$

El predominio de la causa de muerte cardiovascular debida principalmente a progresión de la IC se repite en los registros más recientes, siendo llamativa, en nuestro estudio, la alta incidencia de muertes no cardiacas. A modo de ejemplo, el registro italiano para una población de pacientes ambulatorios con IC crónica, con la misma media de edad, presenta la mitad (16\%) de muertes no cardiovasculares que nuestra serie (35\%). En el mismo sentido, el registro europeo, si bien mostró diferencia en la incidencia de muerte no cardiovascular según los países, en ningún caso superó el 15\%. Por otra parte, nuestro trabajo, al igual que los últimos registros internacionales confirma un franco descenso de las causas de muerte cardiovascular las que abarcaban el $90 \%$ en la década de los $90^{(10,11)}$ 
Si se toma en cuenta el concepto manejado por Orn y Dickstein de que el objetivo de la terapia en pacientes con IC debería ser la prolongación de la supervivencia y el aumento del porcentaje de pacientes que mueren de causas no cardiovasculares, y se analizan datos previos de nuestro grupo, podría interpretarse que este objetivo se habría alcanzado en nuestra población (12-14) De todas maneras, no se debe perder de vista el cambio del fenotipo de la IC en las últimas dos décadas, presentándose pacientes de mayor edad, con mayores comorbilidades y más medicados por lo que tal afirmación no se puede sustentar solamente con los datos de este estudio. ${ }^{(15-17)}$

En nuestro estudio no se halló asociación de variables como la edad, la FEVI, la natremia, diabetes o fibrilación auricular con alguna causa de muerte. Estas variables son predictores de mortalidad en distintos registros y estudios ${ }^{(3,18-20)}$ pero debe tenerse en cuenta que nuestro estudio no analizaba asociación con la mortalidad sino con las causas de muerte. Es probable que esta ausencia de asociación pueda estar determinada por la alta prevalencia de comorbilidades tanto en la población con muerte cardiaca como en aquella con muerte no cardiaca, sumado esto al bajo número de la población estudiada.

Respecto a la clase funcional, si bien no alcanzó niveles de significancia estadística, se muestra una tendencia en la asociación de la peoría de clase funcional con la muerte cardiaca de manera tal que por cada punto de aumento de la clase funcional podría aumentar una vez y media la posibilidad de morir de causa cardiaca. Quizá lo más relevante sea la asociación negativa de cáncer con las causas de muerte cardiacas y positiva con las no cardiacas lo cual podría interpretarse, erróneamente, como un papel protector de la neoplasia en el primer caso siendo, en realidad, expresión del peso importante que esta comorbilidad tiene para determinar la causa de muerte. Esto último toma relevancia a la luz de los datos aportados por Hasin y cols que muestran que pacientes con IC tuvieron mayor riesgo de padecer cáncer en comparación con un grupo control y que a su vez tuvieron mayor riesgo de morir en comparación a pacientes portadores de IC sin cáncer; esto podría vincularse al mayor control y solicitud de pruebas diagnósticas que tienen estos pacientes, incluyendo pruebas de tamizaje de cáncer de diferentes tipos, a lo que se suma que algunos de los factores de riesgo cardiovascular son comunes para el desarrollo del cáncer. ${ }^{(21)}$

Las fortalezas de este estudio están dadas por tratarse del registro de una cohorte seguida estrictamente por un equipo especializado y el hecho de requerirse el máximo grado de certificación (certificado de defunción nacional) para la causa de muerte.

Dentro de las limitaciones del estudio debe considerarse el hecho de tratarse de una cohorte abierta, dinámica y no contar con un registro nacional lo que determina que las causas de muerte de aquellos pacientes que no fallecen en el área de dominio del equipo de seguimiento deban ser rastreadas de forma activa. En el mismo sentido el número de pacientes perdidos de vista, así como la pérdida de datos parciales de algunos pacientes y el desconocimiento de la causa de muerte en 20 pacientes deben ser considerados como posibles sesgos de información y, por último, debe considerarse la posible influencia de los llamados códigos basura en los certificados de defunción que puedan determinar falsos datos.

\section{Conclusiones}

En esta primera publicación que describe las causas de muerte en una cohorte de pacientes con IC en el Uruguay la tasa de mortalidad anual fue 5,6 \% asemejándose a los registros europeos. Se halló una alta incidencia de muertes no cardiacas y asociación de mayor presencia de neoplasia con estas.

\section{Bibliografía}

1- Ambrosy AP, Fonarow GC, Butler J, Chioncel O, Greene SJ, Vaduganathan M, et al. The global health and economic burden of hospitalizations for heart failure: lessons learned from hospitalized heart failure registries. J Am Coll Cardiol. 2014;63(12):1123-33.

2- Ziaeian B, Fonarow GC. Epidemiology and aetiology of heart failure. Nat Rev Cardiol. 2016;13(6):36878.

3- Henkel DM, Redfield MM, Weston SA, Gerber Y, Roger VL. Death in Heart Failure: A Community Perspective. Circ Hear Fail. 2008 May 28;1(2):91-7.

4- Maggioni AP, Dahlström U, Filippatos G, Chioncel O, Crespo M, et al. EURObservational Research Programme: The Heart Failure Pilot Survey (ESC-HF Pilot). Eur J Heart Fail. 2010 Oct;12(10):1076-84. 
5- Curto S; Cavallero M. Morbilidad y Mortalidad por enfermedades cardiovasculares en el Uruguay 2013 - 2014 [Internet]. 2016 [acceso: 30/08/2017]. Available from: http://www.cardiosalud.org/files/ documents/morbilidad_2013_y_mortalidad_2014.pdf

6- ICD-10 Version:2014 [Internet]. [acceso:18/02/2019]. Available from: http://apps.who.int/classifications/ icd10/browse/2014/en

7- Ciapponi A, Alcaraz A, Calderón M, Matta MG, Chaparro M, Soto N, et al. Carga de enfermedad de la insuficiencia cardiaca en América Latina: revisión sistemática y metanálisis. Rev Española Cardiol. 2016 Nov;69(11):1051-60.

8- Pons F, Lupón J, Urrutia A, González B, Crespo E, Díez C, et al. Mortalidad y causas de muerte en pacientes con insuficiencia cardiaca: experiencia de una unidad especializada multidisciplinaria. Rev Española Cardiol. 2010 Mar 1;63(3):303-14.

9- Maggioni AP, Dahlström U, Filippatos G, Chioncel O, Leiro MC, Drozdz J, et al. EURObservational Research Programme: Regional differences and 1-year follow-up results of the Heart Failure Pilot Survey (ESC-HF Pilot). Eur J Heart Fail. 2013;15(7).

10- SOLVD Investigators; Yusuf S, Pitt B, Davis CE, Hood WB, Cohn JN. Effect of Enalapril on Survival in Patients with Reduced Left Ventricular Ejection Fractions and Congestive Heart Failure. N Engl J Med. 1991 Aug 1;325(5):293-302.

11- MERIT-HF Study Group. Effect of metoprolol CR/XL in chronic heart failure: Metoprolol CR/ XL Randomised Intervention Trial in Congestive Heart Failure (MERIT-HF). Lancet. 1999 Jun 12;353(9169):2001-7.

12- Orn S, Dickstein K. How do heart failure patients die? Eur Hear J Suppl Hear J Suppl. 2002;4(4):59-65.

13- Alvarez Rocha P, Ormaechea G, Pouso J, Silvera G, Parma G, Andrade R. Diez años de experiencia en la prescripción de betabloqueantes en la Unidad Multidisciplinaria de Insuficiencia Cardíaca (UMIC) del Hospital de Clínicas de Montevideo. Rev Urug Card. 2015;30: 19-31.

14- Alvarez Rocha P, Ormaechea G, Ricca R. Unidad de Insuficiencia Cardíaca. Breve reseña del tema y evaluación de Gestión de la Primera Unidad de Insuficiencia Cardíaca Pública del Uruguay (grupo UMIC). Arch Med Int. 2009; 31:11-17.

15- Wong CY, Chaudhry SI, Desai MM, Krumholz HM. Trends in Comorbidity, Disability, and Polypharmacy in Heart Failure. Am J Med. 2011 Feb 1;124(2):136-43.

16- Montero Pérez-Barquero M, Conthe Gutiérrez P, Román Sánchez P, García Alegría J, Forteza-Rey J. Comorbilidad de los pacientes ingresados por insuficiencia cardiaca en los servicios de medicina interna. Rev Clínica Española. 2010;210(4):149-58.

17- Adams KF, Fonarow GC, Emerman CL, LeJemtel TH, Costanzo MR, Abraham WT, et al. Characteristics and outcomes of patients hospitalized for heart failure in the United States: rationale, design, and preliminary observations from the first 100,000 cases in the Acute Decompensated Heart Failure National Registry (ADHERE). Am Heart J. 2005 Feb;149(2):209-16.

18- Derfler MC, Jacob M, Wolf RE, Bleyer F, Hauptman PJ. Mode of Death From Congestive Heart Failure: Implications for Clinical Management. Am J Geriatr Cardiol. 2004 Nov 1;13(6):299-304.

19- Bocchi EA. Heart failure in South America. Curr Cardiol Rev. 2013 May;9(2):147-56.

20- Mehta PA, Dubrey SW, McIntyre HF, Walker DM, Hardman SMC, Sutton GC, et al. Mode of death in patients with newly diagnosed heart failure in the general population. Eur J Heart Fail. 2008 Nov $1 ; 10(11): 1108-16$.

21- Hasin T, Gerber Y, McNallan SM, Weston SA, Kushwaha SS, Nelson TJ, et al. Patients With Heart Failure Have an Increased Risk of Incident Cancer. J Am Coll Cardiol. 2013 Sep;62(10):881-6.

\section{Aporte cada autor al trabajo}

Silvana Estrada Escobar: concepción y diseño del trabajo, recolección de datos, análisis e interpretación de los datos, redacción del manuscrito.

Pablo Álvarez Rocha: concepción y diseño del trabajo, recolección de datos, análisis e interpretación de los datos, análisis estadístico, redacción del manuscrito.

Lucía Florio Legnani: análisis e interpretación de los datos, análisis estadístico, redacción del manuscrito.

Gabriela Ormaechea Gorricho: concepción y diseño del trabajo, análisis e interpretación de los datos, revisión crítica del manuscrito. 